\title{
Filosofia, gênero e ciência: entrevista com Eulalia Pérez Sedeño
}

\author{
Cristina Tavares da Costa Rocha \\ Universidade Federal de Santa Catarina \\ Miriam Pillar Grossi \\ Universidade Federal de Santa Catarina
}

Pioneira nos Estudos de História e Filosofia da Ciência e Gênero, com trajetória acadêmico-científica de destaque, destreza no raciocínio, capacidade singular de atenção a outrem, visão de vanguarda no conhecimento, além de simpatia contagiante, Eulalia Pérez Sedeño reúne características únicas para estar na gênese, e ser uma das principais articuladoras ibero-americanas, do campo interdisciplinar dos Estudos de Gênero, Ciência e Tecnologia. A partir de sua iniciativa, foi criado o Congreso de Ciencia, Tecnología y Gênero, que se realiza alternadamente em países da América Latina e na Espanha, sendo que a próxima edição acontecerá em 2010 em Curitiba (Brasil).

Eulalia é catedrática de Lógica e Filosofia da Ciência e pesquisadora no Departamento de Ciência, Tecnologia e Sociedade do Instituto de Filosofia do Conselho Superior de Investigações Científicas (CSIC), o principal organismo público de pesquisa na Espanha.'

Seu currículo é extenso e qualificado. Foi diretora da Fundação Espanhola para a Ciência e Tecnologia (FECYT, 2006-2008) e integra o Conselho Assessor da Rede Cátedra de Mulheres, Ciência e Tecnologia na América Latina desde outubro de 2005. Foi vice-presidente da Associação das Mulheres Investigadoras e Tecnólogas (AMIT) e presidente da Sociedade de Lógica, Metodologia e Filosofia da Ciência na

Copyright $\odot 2008$ by Revista Estudos Feministas

1 O CSIC participa ativamente na política científica de todas as comunidades autônomas da Espanha, através de seus diversos centros agregados por oito áreas tecnocientíficas. 
Espanha. Pertence ao Conselho Editorial da Fundação Carolina. Organiza mensalmente o seminário aberto ao publico "Las Mujeres también Investigan" (FECYT e Biblioteca Nacional da Espanha), com participação de cientistas e personalidades de reconhecido prestígio em seu âmbito profissional, da Espanha e de outros países.

Atuou em vários órgãos governamentais, como a Agência Nacional de Avaliação e Prospectiva (ANEP), Ministério da Educação e Ciência (MEC), Fundação Européia de Ciência (ESF), Secretaria de Estado de Igualdade do Ministério do Trabalho e Assuntos Sociais, e pertence a comitês editoriais de revistas espanholas e internacionais. Foi professora e pesquisadora nas Universidades de Barcelona, Complutense de Madrid, Cambridge (Inglaterra), Califórnia, em Berkeley (USA), Universidade de São Paulo, dentre outras.

Autora e editora ou coeditora de diversos livros, publicou mais de 70 artigos científicos na área, dentre outros: "Igualdad y equidad en ciencia y tecnología: el caso iberoamericano", Arbor, v. CLXXXIV, n. 733, 2008; e "Sexos, géneros y otras especies: diferencias sin desigualdades", en El segundo escalón. Equilibrios y desequilibrios de género en ciencia y tecnología, Sevilla: ArCiBel, 2006.

Esta entrevista foi realizada por ocasião do $100^{\circ}$ Congresso Internacional Interdisciplinar Mundos de Mulheres (Women's World 08), ocorrido na Universidad Complutense de Madrid, na Espanha, de 3 a 9 de julho de 2008, em seu atual local de trabalho, o CSIC.

Eulalia, nascida no Marrocos, de pais espanhóis, tem quatro irmãs; está casada com um filósofo da linguagem, com quem teve dois filhos. Nesta entrevista ela narra sobre sua trajetória no campo acadêmico-científico, sua posição institucional no campo da filosofia espanhola e no topo da hierarquia da FECYT. Por ter sido uma das poucas mulheres de sua geração a ter rompido o "teto de vidro", suas pesquisas refletem uma instigante visão sobre as conquistas e as lacunas no campo dos estudos de gênero. 
2 Las notas de pié de página, tanto las que hacen referencias a las personas mencionadas por la entrevistada, cuanto as que se refieren a conceptos, son de responsabilidad de las entrevistadoras.

Revista Estudos Feministas: Hable sobre su trayectoria intelectual de filósofa feminista. ${ }^{2}$

Eulalia Pérez Sedeño: Siempre me he considerado feminista. Pero me dediqué, en un inicio, a la Filosofía, y dentro de Filosofía me especialicé en el campo de la Lógica y de la Filosofía de la Ciencia. Y en una época en la que todavía no creíamos que los valores intervienen en la ciencia y en la tecnología. Hice mi tesis de licenciatura sobre un sistema lógico-matemático, libre de supuestos existenciales.

REF: ¿En qué universidad lo hizo?

EPS: Fue en la Universidad Autónoma de Madrid (UAM). Luego por varios motivos personales fundamentalmente - es que mi mentor y maestro, Alfredo Deaño, murió, ${ }^{3}$ y además porque pensé que aquello ya no me daba mucho más - me pasé a la Historia de la Ciencia. Debo decir que en principio, siempre me interesó la Historia de la Ciencia, porque a mí me gustó siempre mucho la Historia. Y en la Historia de la Ciencia tuve un profesor excelente en la Licenciatura, que dejó la universidad por motivos políticos. Cuando yo acabé la carrera de Filosofía en la Universidad Autónoma de Madrid, a través de Alfredo Deaño se me ofrecieron un puesto como profesora contratada ayudante en la Universidad de Barcelona. Es así que yo ya era entonces profesora de Lógica y Filosofía de la Ciencia en la Universidad de Barcelona.

REF: ¿En qué año fue eso?

EPS: Acabé mis estudios en el año de 1976 y en 1977 me fue a trabajar en la Universidad de Barcelona donde estuve hasta 1983. Salí para hacer la tesis doctoral que iba a ser, en principio, un análisis lógico de una teoría científica, en concreto de la astronomía copernicana. Pero fui retrocediendo y, al final, acabé haciendo una tesis sobre la Historia de la Astronomía Antigua, o sea, sobre el papel de la teoría, de la experiencia y de la observación en la Astronomía Antigua. Lo que yo planteaba era: lo que nos ha llegado de la Astronomía Antigua es la tradición de los modelos cosmológicos desde Anaximandro, Anaxímenes, Tales, Platón, Eudoxo, Leucipo, Aristóteles, Ptolomeo. Pero cuando estudias Ptolomeo, te das cuenta de que has tenido que haber un trabajo de observación muy importante. Pues yo lo que hice fue estudiar toda una tradición que no se conoce

${ }^{4}$ Hacen pronóstico meteorológico por medio de las posiciones de los planetas y las estrellas. mucho, que es más observacional, de los parapegmatistas, ${ }^{4}$ que son los astrónomos que hacen observaciones para establecer ciclos lunares y solares precisos. (...) Hay ahí toda una tradición que surge en el siglo VIII a.n.e. Ya aparece en la obra de Hesiodo "Los trabajos y los días", donde hay una parte muy bonita donde se va diciendo qué trabajos hay que hacer, en qué momento del año y con qué momentos astronómicos coinciden.Y luego sigue con Anaximandro, pero también con 
${ }^{5}$ Sir Geoffrey Ernest Richard Lloyd, historiador británico, nació en Swansea (Gales) en el año 1933. Uno de sus diversos libros es: Ancient Worlds, Modern Reflections: Philosophical Perspectives on Greek and Chinese Science and Culture. New York: Oxford Univ. Press, 2004. En 1997 se le concedió el título de "Sir" en reconocimiento a su contribución en la "historia del pensamiento". Este es uno de los autores que ha estudiado las ideas sobre la biología de la mujer, por ejemplo su libro Science, Folklore and ldeology: Studies in the Life Sciences in Ancient Greece. Cambridge: CUP, 1983. Se casó con Janet Lloyd, que ha trabajado como traductora para Shell y con quien tuvo 3 hijos. Actualmente, vive gran parte del año en su residencia en España, donde se dedica a la escritura.

6 Janet Lloyd es traductora de 60-70 libros; ganó el premio ScottMoncrieff por dos veces, en 1978 y 1997. Uno de varios libros que ha traducido: Arms and the Woman: Just Warriors and Greeck Feminist Identity.

${ }^{7}$ De ese encuentro nace la obra Conceptualización de lo femenino en la filosofía antigua, con coordinación de Eulalia Pérez Sedeño y presentación de Celia Amorós, publicado en 1994, por Siglo XXI de España Editores, S.A. En ese libro hay un capítulo de Eulalia Pérez Sedeño con el título: "Masculino y femenino en la cosmología de Ptolomeo" (p. 91-111).
Eudoxo, con Calipo hasta llegar, claro, a Ptolomeo. Esa fue mi tesis.

\section{REF: ¿En la UAM?}

EPS: Sí. En la Universidad Autónoma de Madrid, no sé si fue en 1984 o 1985. Un poco antes, en 1983, me ofrecieron un trabajo en la Universidad Complutense de Madrid (UCM) y me volví como profesora a la UCM, donde luego saqué una plaza definitiva. Y cuando la saqué, en el año de 1986, yo ya tenía un hijo de 5 años.

Pero siempre me había querido ir fuera. Bueno, primero la tesis de mi marido, nuestro hijo, la tesis mía, la oposición... Cuando ya estábamos todos más o menos tranquilos decidimos ir fuera de España. Me puse en contacto con un profesor, Sir Geoffrey Lloyd ${ }^{5}$ de la Universidad de Cambridge, una autoridad en los estudios clásicos. Él me acogió con los brazos abiertos y me puso en contacto con otro profesor, un historiador de la Astronomía. No obstante, mi idea al irme a Cambridge era que después de haber estudiado la Astronomía Antigua, tenía que estudiar Medicina, porque pensaba - y tengo escrito alguna cosa sobre eso - que, aunque son disciplinas diferentes, hay problemas y métodos similares pero también distintos, y me interesaba hacer una comparación entre ellas. Estuve en Cambridge en 1988.

Al empezar a trabajar sobre la Medicina, y como yo hablaba mucho sobre los derechos de las mujeres con Geoffrey y su mujer, ${ }^{\circ}$ que es una mujer estupenda, un día me planteé un problema muy bonito e interesante con respecto a la antigüedad y a las mujeres, que da origen a mis estudios de género. He creído que interesa a la cuestión feminista. El problema es que, por razones del pudor, los médicos no pueden explorar a las mujeres. Pero, políticamente, legalmente, las mujeres son menores legales; carecen de autoridad. Por lo tanto, el médico tiene que fiarse de la autoridad de la paciente cuando ésta le cuenta lo que tiene. Pero a la vez, hay toda una serie de mujeres que intermedian entre el médico y la paciente, como son parteras, curanderas etc. Yo me planté esa cuestión y empecé a trabajar sobre ella.

La puntilla fue cuando, de vuelta a España, Celia Amorós me dijo que quería hacer una reunión sobre género o feminismo y antigüedad y me pidió que hiciera algo. ${ }^{7}$ Como yo había empezado a pensar sobre eso, dije: "Conozco muy bien la Astronomía tolemaica, pero sobre género y astronomía tolemaica ... a ver".

REF: ¿En qué año ocurrió su vuelta a España y su contacto con Celia Amorós?

EPS: Volví a España a finales del 1988 y tuve mi segundo hijo en 1989. También en ese año tuve contacto con Celia Amorós. Yo 
${ }^{8}$ ARBOR: Ciencia, Pensamiento y Cultura, Madrid, tomo CXLIV, n. 565 , enero 1993. Número especial Mujer y Ciencia. Comp. Eulalia Pérez Sedeño.

${ }^{9}$ Evelyn Fox Keller es catedrática de Historia y Filosofía de la Ciencia en el Massachussets Institute of Technology (MIT); es una de las investigadoras más conocidas dentro del movimiento CTS (ciencia, tecnología y sociedad) por sus estudios sobre género y ciencia.

${ }^{10}$ El 16 de mayo de 2007, Evelyn Fox Keller fue la protagonista del ciclo "Las mujeres también investigan" que organizan la FECYT y la Biblioteca Nacional de España.

1 Filósofa, docente en la Universidad de Buenos Aires (UBA) y en la Universidad de Rosario. Ha defendido su tesis: "Género, subjetividad y conocimiento". Es investigadora en el Instituto Interdisciplinar de Estudios de Género en Ia UBA. Es directora académica del Instituto Hannah Arendt. De entre sus libros, hay: Sexualidades migrantes: género y transgénero (Feminaria Editora) y Sexualidad represora, presentado en la Feria del Libro de 2008, en Argentina. Ha sido elegida diputada en Buenos Aires, de 2007 a 2011.

12 Profesora Adjunta de la Cátedra de Neurología de la Facultad de Medicina de la UBA y directora del Centro de Epilepsia, que funciona en la División de Neurología del hospital Ramos Mejía. Además es investigadora del Consejo Nacional de Investigaciones Científicas y Técnicas (Conicet) sobre Clínica y Diagnóstico de la Epilepsia.

${ }^{13}$ Catedrática de Medicina en la UBA. Ha publicado diversos textos en el área.

14 Será en la Universidad Tecnológica Federal del Paraná (UTFPR), bajo la coordinación de Marilia Gomes de Carvalho. ya la conocía, porque éramos colegas. Luego en seguida, publiqué el famoso número de Arbor. Creo que es el primero que se edita en la España (en español también) sobre género y ciencia, que es un número especial que está agotadísimo; salió en el año de 1993, pero lo preparamos antes. Llámase: Mujer y Ciencia. ${ }^{8}$ Fue ahí que todo empezó. En el año siguiente, deberíamos otra vez salir y esta vez eligió mi marido a dónde ir. El es profesor en la Universidad Nacional de Educación a Distancia. Es filósofo del lenguaje. Él eligió Berkeley porque quería trabajar sobre metáfora. A mí me pareció muy bien porque allí estaba Evelyn Fox Keller. ${ }^{9}$ Pero cuando llegué allí ella ya se había ido. No la llegué a conocer entonces. En 2007, ella estuvo aquí en CSIC porque la invitamos a dar un seminario. ${ }^{10}$ Yo se la comenté: "iYo me fui a Berkeley para estar contigo pero tú te habías ido cuando llegué!" Y así íbamos construyendo nuestro camino.

\section{REF: ¿Cuando ha llegado usted a la ciencia moderna?}

EPS: Me gustaba mucho hacer recuperación de la historia de mujeres científicas. Escribí algunos artículos sobre astrónomas, sobre matemáticas, tengo alguna cosa sobre mujer y medicina en la clase médica. Empecé con eso y luego llegué al que es conocido hoy como estudios de la ciencia, tecnología y género. Cuando yo volví de Berkeley en octubre de 1995, una cosa importante, creo, fue que a mí se me ocurrió la idea de los congresos de ciencia, tecnología y género, porque yo había estado haciendo muchas cosas e igual que yo había que haber más gente en España haciendo algo sobre ciencia y género. Yo, aún estando en Berkeley, vine a un congreso en Barcelona. Allí conocí algunas personas que hacían algo de ciencia y género y que estaban empezando en esos momentos. Pensé: "¿por qué no organizamos algo para reunirnos todas?" Entonces lanzamos ese $1 .{ }^{\text {er }}$ congreso, que fue realizado en la Universidad Complutense de Madrid. Tuvimos ayuda del Ministerio de Asuntos Sociales por medio del Instituto de la Mujer. Lo divertido fue que yo quería hacer un congreso español, pero se convirtió en nivel iberoamericano porque aparecieron unas mexicanas y unas argentinas. Estas últimas eran Diana Maffía, ${ }^{11}$ Silvia Cochen ${ }^{12}$ y Ana Franchi ${ }^{13}$ y dijeron: "Esto es genial. El próximo lo organizamos nosotras." Y así empieza todo eso. El siguiente fue en Buenos Aires, en 1998. De una manera muy informal acordamos hacerlo cada dos años, y que cada vez fuera a un lado del Atlántico. En 2000, fue en Panamá, porque había una panameña en Buenos Aires que nos convenció de que seria bueno para posicionar sus estudios en Panamá y además era el año en que se les devolvía el Canal. En el año siguiente volvió a ser en Madrid; lo organizamos con Marta González, Concha Roldán y con más personas. En 2004 fue en México. En 2006 en Zaragoza. En 2008 en Cuba, Habana. Y 2010 será en Curitiba/Brasil. ${ }^{14}$ 
Entremedia lo que pasó fue que en 1999 saqué la cátedra de Lógica y Filosofía de la Ciencia en la Universidad del País Vasco. Debo decir que fue una cátedra estupenda.

REF: ¿Cómo se dio ese proceso?

EPS: Es algo completamente extraño. Es muy raro. Seguramente, el $99 \%$ de las personas que son, en España, profesores de la universidad o investigadores del Consejo Superior de Investigaciones Científicas han pasado como máximo por dos instituciones, no por más. Al menos eso es lo normal. Pues yo estaba en la Universidad Complutense de Madrid. Y había algo muy extraño. Es que no había ninguna catedrática de Lógica y Filosofía de la Ciencia. Entonces, se convocó una plaza para cubrir una cátedra de Lógica y Filosofía de la Ciencia en la Universidad de País Vasco. Las cátedras tienen lo que se llama denominación de área, pero luego llevaban un perfil en el caso de la cátedra del País Vasco. Ese perfil era muy divertido porque ponía que había que tener docencia e investigación en: Lógica, Historia de la Ciencia y Filosofía de la Ciencia. Creo que en esos momentos la única persona en el país que tenía las 3 áreas era yo. Había hecho mi tesina sobre Lógica e Historia de la Ciencia; además, mi tesis sobre la Ciencia y mi docencia y publicaciones y los proyectos de investigación que había dirigido abarcaban la lógica, la historia y la filosofía de la ciencia. Antes las cátedras las juzgaba un tribunal, donde había dos personas propuestas por la propia universidad y tres personas que se sorteaban entre todos los catedráticos.

Pensé: “iPues me voy a presentar!", porque no podía desanimar mucha gente, sobretodo las mujeres; aquellas de mi área y de mi entorno me decían que yo tenía que presentarme porque era una vergüenza que no hubiera mujeres catedráticas en el área y que si había alguien que tenía esa posibilidad, esa era yo. Pues como soy un poco lanzada para esas cosas... No decidí presentarme hasta que no vi el tribunal. Pero cuando vi el tribunal que salió por sorteo pensé que tenía posibilidad.

REF: ¿Cómo es eso? ¿Se presenta un conjunto de trabajos o hay una prueba?

EPS: Hay dos ejercicios. Hay una primera parte donde se presenta el curriculum y un plan docente-investigador. En esta parte no tienes tiempo límite y te pueden preguntar todo lo que se quiera del área. El segundo ejercicio lo que se hace es presentar un trabajo de investigación que puede ser propio o puede ser del grupo de investigación al que se pertenece. Pero ya eso ha cambiado. Pero pensaba que no la sacaría. Me daba igual. Así mismo, pensé en darme un gustazo de presentar sobre lo que yo estaba trabajando entonces, mi investigación sobre Ciencia y Género que era sobre los valores de género en 
la investigación científica. Pues, sorprendentemente, la saqué. Tuve que ir al País Vasco. Dejé a mi familia en Madrid. A los 3 años fue cuando el presente Consejo Superior de Investigaciones Científicas (CSIC) contactó conmigo sobre una serie de cuestiones sobre la situación de las mujeres en el CSIC y el caso es que me ofreció venir al Consejo en Comisión de Servicios. Esto significa que sigues manteniendo tu plaza en mi caso, la cátedra en el País Vasco, pero la institución que te acoge te paga el sueldo para que tú estés trabajando para dicha institución. Entonces, me vine al CSIC, donde el Instituto de Filosofía me acogió con los brazos abiertos, y después de estar 3 años en comisión de servicio pues decidieron solicitar que se sacara una plaza para mí. Entonces, yo salí...

Cuando hubo la posibilidad de sacar una plaza de profesor de investigación, que equivale a una de catedrático en el Consejo, estuvimos discutiendo también con qué denominación se sacaba la plaza. Si en Filosofía de la Ciencia, si en Historia y Filosofía de la Ciencia. Por supuesto toda la gente, amigas, colegas, que me conocían, decían: "¿Ciencia, tecnología y género? Pero no van a querer eso, y tal..." Y ahí mi marido me ayudó mucho porque me dijo que me diera el gustazo de sacarla como "ciencia, tecnología y género". Además ahí hay que decir que el Presidente del Consejo, que era quien tenía que decidir, pues optó por algo nuevo e innovador, como ciencia, tecnología y género, al revés de algo más clásico como era el de Filosofía de la Ciencia. Entonces, soy ahora profesora de investigación en Ciencia, Tecnología y Género.

REF: ¿En qué año fue eso?

EPS: Llegué en Comisión de Servicio en noviembre de 2002. En noviembre de 2005 hice la oposición, porque también tuve que volver a hacer la oposición para ingresar aquí [en CSIC], para tener la plaza definitiva. Pero en realidad tomé posición en junio de 2006. En octubre de 2006 me nombraran directora general de la FECYT (Fundation Española de Ciência Y Tecnología).

REF: ¿Cómo es esa nominación?

EPS: Esa nominación es puramente política. O sea, el Secretario de Estado que es el Presidente del Patronato de la Fundación, nombra a alguien de su confianza para desarrollar las políticas que a él le parecía que tenía que hacer la Fundación. Él a mí me llamó y me dijo: "Quiero que la Fundación se convierta en el organismo que desarrolle la cultura científica en España". Y me eligió para la dirección general. Yo era la que tenía un poco de reparo, pero la gracia es que me dijo que tenía la posibilidad de llevar a la práctica muchas de las cosas que en teoría yo estaba diciendo, porque claro yo trabajo sobre ciencia, tecnología y género pero eso está en la ciencia, tecnología y 
sociedad. Se trataba de incrementar las relaciones entre ciencia, tecnología y sociedad y con dinero para hacerlo. Cuando llegué a la Fundación tenía 12.500.000 • (doce millones y medio) de euros de presupuesto y cuando me he ido la he dejado con 24.500 .000 (veinte y cuatro millones y medio) de euros, sin contar el presupuesto destinado a los contratos postdoctorales que también gestiona FECYT. LA FECYT, es una Fundación pública, porque todo el dinero viene de los presupuestos generales del Estado.

REF: ¿Cuánto tiempo se quedó usted? ¿Cómo fue su experiencia?

EPS: Casi dos años. Creo que ha sido una experiencia muy buena, muy enriquecedora. Primero, porque he aprendido muchísimo, de muchas cosas muy diferentes, porque la Fundación es bastante compleja. No tiene sólo la parte de la cultura científica. Tiene también una parte de infraestructura. FECYT maneja, coordina y paga el acceso en toda España de la Web of Knowledge, por ejemplo. Pero también hemos coordinado toda la política científica del país, o sea, todo el establecimiento de un nuevo plan nacional de ciencias y tecnología. Mi proyecto dentro del Ministerio de Educación y Ciencia, fue acercar la ciencia a la sociedad, poner en marcha estructuras estables de comunicación científica, incluyendo un servicio de información científica (SINC). Entonces, creo que he aprendido mucho, ha sido muy enriquecedor; también ha sido muy interesante porque es una Fundación pública pero funciona como una empresa privada.

Ese aspecto yo no lo conocía y si a mí me hubieran dicho hace años que iba a ser una empresaria, "iQue, ni loca!" Pues he tenido que gestionar contratos, decidir a quién contrataba, quién entraba, tuve que tomar alguna decisión compleja y complicada en ese sentido. Porque he visto lo que es ser mujer ante determinadas personas que pensaban que podían manejarme. Entonces, tienes que tomar decisiones porque si dejas de tomarlas en el inicio, en seguida... por ejemplo, hay un caso, una cosa que jamás imaginara: que una persona que no podía firmar determinados papeles, los firmó, cuando lo sabía y nunca lo había hecho antes. Entonces yo lo interpreté como que estaba marcando el territorio, ya está. Yo tuve que marcar más.

REF: Y de todo, ¿eso fue lo más difícil para usted en términos de género?

EPS: Eso ha sido difícil. Pero ha sido más difícil y terrible ver que hay mujeres que no reconocen la autoridad femenina. Además el caso concreto de algunas mujeres relativamente cercanas no íntimas, pero no personas manifiestamente extrañas. Más que decepcionar también ha dolido. Esto es: darte cuenta de 
que estás con una persona que sabe que eres su superior y hace más caso al otro que no lo es. ¿Por que? Ah, es por que es varón/hombre. No es lo mismo tener la teoría que verlo en la práctica con una misma.

REF: Usted escribió sobre la endogamia en la academia española y su relación a las mujeres académicas. ¿Cómo ve usted su carrera?

EPS: Yo estudié en la Universidad Autónoma de Madrid y luego trabajé en la de Barcelona, en la Universidad Complutense, en la del País Vasco, y luego en el Consejo, más Berkeley, Cambridge, México, Brasil, donde estuve dos meses y medio en 1991 en la Universidade de São Paulo (USP), dando un seminario sobre mujer y ciencia para el Instituto de Historia de la Química, etc.

Pero no se trata sólo de irse a otro país. Lo importante es que se vaya a otro centro de excelencia. Además también se pueden hacer instancias cortas en el exterior. Por ejemplo: ¿Por qué no te puedes ir 3 meses? Es mucho más fácil dejar a unos hijos 3 meses que dos años. O sea, hay muchas combinaciones posibles empezando por la propia institución para que ayude económicamente para que tú puedas llevar la familia.

Siempre digo que es muy malo que la gente trabaje en el mismo sitio que ha estudiado y el mismo sitio en que ha sido ayudante, luego saca la plaza, y no se mueve para nada. Eso perjudica a las mujeres, porque: ¿Cómo se deciden las plazas que salen de los departamentos? Es en una reunión dónde las mujeres son una minoría. Entonces las que acaban saliendo son para los hombres. ¿Por que me marché yo para ser catedrática a la Universidad de País Vasco? Por una razón muy sencilla. Porque me di cuenta que en la Complutense iba a poder ser catedrática no sé cuando, pero desde luego no inmediatamente. ¿Por qué? Nosotros nos sometíamos a evaluación externa, para ver que personas de la facultad y del departamento tenían un currículo adecuado para ser catedrático. Cuando me sometí, me quedé la tercera de toda la facultad. Y entonces tenía 20 años menos que ahora, claro. Pero tenía mejor currículo que mucha gente que se quedó. Y se computaban que habían tenido puestos de responsabilidad. O sea, la evaluación era el currículo docente, el currículum de investigador y currículum administrativo. Yo tenía muy buena puntuación en los otros, pero no tenía puestos de responsabilidad; no había sido decana ni jefa de departamento, y eso se puntuaba mucho. Ahora eso ha cambiado mucho.

REF: Usted habla de las cosas que ha escrito.

EPS: Estoy muy contenta. Quiero decir, no es que estoy contenta por lo que he escrito, pero hay una reflexión que tuve que hacer. En las oposiciones o concurso para conseguir el puesto de 
15 Doctora en Química por la Universidad Cumplutense de Madrid, es profesora e investigadora científica de Ciencia, Tecnología y Sociedad del Instituto de Filosofía del CSIC. Uno de sus varios textos: "El embarazo tecnificado: diagnóstico y salud prenatales". ${ }^{16}$ Maria Soledad Paloma Alcalá Cortijo es licenciada en Físicas y DEA en Filosofía por la Universidad Complutense de Madrid; ha sido responsable del programa de Igualdad de Oportunidades entre los sexos en la Dirección Provincial de Educación del Ministerio de Educación y Ciencia de Madrid (1989-1993); pertenece a los grupos de expertos: "Ciencia y enseñanza" y "Mujer y ciencia" de la FECYT; Uno de sus diversos artículos: "A ras de suelo. situación de las mujeres en las instituciones científicas", en Ciencia, Tecnología y Género en Iberoamérica. Madrid: CSIC, 2006. p. 89-98. Monografías, n. 29. profesora de investigación en el Consejo, uno de los ejercicios es que hay que contar tu hoja de vida, pero en función de la investigación que has llevado a cabo y de los proyectos del futuro. Entonces, he visto que he sido pionera en España. He tenido que hacer durante mucho tiempo un gran trabajo de divulgación y de promoción. Me acuerdo, por ejemplo, que pasé varios años, a partir de 1988, con una situación: los profesores de Educación Secundaria tenían que hacer cursos de reciclaje y todos los cursos, de la materia que fuera, tenían que llevar un módulo de género. Al final, la persona que hacía eso empezó a echar mano a mí. Pasé dos años o tres que iba a dar muchos módulos sobre los estudios de género, para secundaria, en este sentido de participación, contar un poco sobre todos esos estudios que se habían hecho en las clases entre niños y niñas, los estereotipos, o sea, todo ese tipo de cosas que también en la universidad se dan y mucho. Esto lo cuento para recordar que esta es una unidad que he tenido que hacer mucha difusión y divulgación de estas cuestiones y que me ha quitado tiempo para otras cosas.

Creo que hice una primera parte sobre el papel de las mujeres en las instituciones científicas. Creo que hemos hecho un buen trabajo con el estudio que hicimos para el Ministerio de Educación y Ciencia sobre la situación de las mujeres en el sistema científico y técnico español. Creo que hoy es el único que hay así amplio en España. Luego he hecho cosas complementarias, incluso yo coordinaba junto con María Jesús Santesmases ${ }^{15}$ y Paloma Alcalá ${ }^{16}$ el que hicimos para CSIC/ FECYT en 2004, antes de que yo dirigiera la Fundación, pero no es tan amplio. Cuando llegué [a FECYT] lo actualizamos, y además hemos hecho la traducción al inglés porque no hay nada de esto en Europa ni en España. Y luego está la parte de percepción: de cómo la sociedad española percibe la ciencia y la tecnología, pero con una mirada específica de género. Y está la investigación actual, pues tenemos un proyecto de investigación financiada desde hace 5 años que es sobre las tecnologías de los cuerpos. O sea, parto de la perspectiva de que hay que analizar las tecnologías primero viendo en que consisten, pero también que consecuencias tienen, a quién benefician, a quién perjudican. Sobre cómo afecta a las mujeres en particular. Cuando hablamos de cualquier tecnología, por ejemplo, la energía atómica, es una tecnología que tiene energía pero no la conozco, todavía de alguna forma afecta a las mujeres. También hay otras. Hay un estudio bonito que mencionaba el otro día Carme Alemany, sobre como se diseñan a los artefactos tecnológicos, pues no piensan en quiénes los van a usar.

Nos interesa mucho la reproducción asistida desde que empezó en todo el mundo y también en España. Empezamos esos estudios sobre las tecnologías aplicadas a los cuerpos. Primero, 


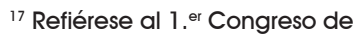
Ciencia, Tecnología y Género, ocurrido en Madrid, en 1996. por la reproducción asistida; seguimos por el diagnóstico prenatal - claro, hay ahí algo que es muy interesante, porque afecta mucho desde cómo se convierten los cuerpos de las mujeres en objetos e incluso objetos científicos, sujetos a la observación hasta el punto que muchas veces se les elimina toda la capacidad de decisión y seguimos, claro, directamente sobretodo a las células troncales.

Luego se nos incorporó un grupo de gente muy interesante que trabaja sobre los cuerpos de los seres intersexuales y transexuales. Este año estoy ya vendo sobre cirugías reparadoras, sobretodo las tecnologías de las cirugías estéticomamaria. Es lo que vamos a hacer. Es evidente la manipulación del cuerpo para ajustar al patrón que nos marcan la moda, la estética, etc., pero tiene también esta otra parte de la cirugía reparadora ante un cáncer mamario, o lo que sea. Hay sus implicaciones estéticas, éticas, y, por supuesto, todas las implicaciones sociales también.

Y ahí lo que decía: seguramente porque he tenido que estar haciendo todas esas cosas, he publicado menos de lo que me gustaría. Pero, tenemos dos proyectos muy inmediatos, que vamos sacar un libro conjunto de todo el equipo sobre tecnologías de los cuerpos, que esperamos para el próximo semestre.

REF: ¿Cómo ve usted y cuándo empiezan los estudios de género en España? ¿Vienen de los movimientos del feminismo o era algo más teórico? ¿Y los aspectos de investigación? ¿Y los aspectos políticos?

EPS: Hay ahí muchas cosas. Da para hacer una tesis. Vienen de los movimientos, pero también es teórico.

REF: Cuando cuenta sobre la génesis de su preocupación por el género en aquella época ¿ya eran estudios de género o estudios de mujer? ¿O teoría feminista?

EPS: Creo que empezamos a usar género a partir del año 1993. Creo que el número de Arbor se llama todavía Mujer y Ciencia. Ni siquiera en plural. Es que era un problema que entonces no nos planteábamos. Creo que desde el congreso ${ }^{17}$ ya se llama género. Esto es 1996. Creo que en España empezamos un par de años antes, al volver de Estados Unidos. Al llegar de Estados Unidos empecé a hablar de género. Pero es sobretodo una estrategia política y académica, porque eso te da cierta lógica. Y lo sigo viendo como tal. En España, sobretodo desde 1996, cuando entra el Partido Popular, hay una especie de rechazo del feminismo, pero ahora se está pasando. O sea, el discurso de muchas mujeres, incluso las que estabas viendo que hacían cosas "feministas" empezaba diciendo: "Yo no soy feminista, pero..." Y a continuación decían lo que querían. 
${ }^{18}$ Catedrática de Filosofía Moral y Política de la Universidad Nacional de Educación a Distancia (UNED). Es autora del libro Sexoy filosofía; Del miedo a la igualdad, de entre otros. Nació en 1950, es casada con filósofo y tiene una hija que también es filósofa.

${ }^{19}$ Catedrática de Filosofía Moral y Política en la Universidad Nacinal de Educación a Distancia (UNED). Publicó, de entre otros, el libro La gran diferencia y sus pequeñas consecuencias. Para la emancipación de las mujeres. Madrid: Cátedra, 2004. Colección Feminismos.

${ }^{20}$ Feminismo de la igualdad: en una sociedad los hombres y las mujeres tienen los mismos derechos y obligaciones. Las mujeres seguían los conceptos del feminismo socialista. Disponible en: http:// www.fmujeresprogresistas.org/ feminismo4.htm. Acceso en: jul. 2008.

${ }^{21}$ Feminismo de la diferencia: concebía la necesidad de que se entendiera a las mujeres no sólo como militantes, sino como totalidad, como personas. No podía hacerse separaciones tajantes entre lo privado y lo público. Disponible en: http:// www.fmujeresprogresistas.org/ feminismo4.htm. Acceso en: jul. 2008.

22 Eulalia se refiere al $10^{\circ}$ Congreso Mundos de Mujeres/ Women's Worlds 2008: La igualdad no es una utopía: Nuevas fronteras, avances y retos. Está se refiriendo más específicamente a la mesa-redonda intitulada La construcción de género en la tecnología. Ese congreso tuvo lugar en la Universidad Complutense de Madrid, del 3 al 9 de julio de 2008, en Madrid, España.

${ }^{23}$ Flora de Pablo fue presidenta de la Asociación de Mujeres Investigadoras y Tecnólogas (AMIT). Ha recibido el premio Carmen de Burgos. Es doctora en Medicina, profesora e investigadora del Centro de Investigaciones Biológicas del
Los estudios de ciencia, tecnología y género, o ciencia y género parece que era una manera de legitimarlos en el sentido de que estaban bien establecidos en Inglaterra y Estados Unidos. Con respecto al feminismo, creo que lo fundamental es que aquí tenemos dos grandes teóricas del feminismo que son Amelia Valcárcel ${ }^{18}$ y Celia Amorós. ${ }^{19} \mathrm{Y}$ además es de decir que son del feminismo de la igualdad..$^{20} \mathrm{O}$ sea, seguramente, por haberme dedicado a la ciencia, el feminismo de la diferencia ${ }^{21}$ no me ha interesado demasiado. Porque la diferencia ha sido la que se ha utilizado para mantenernos en un estado de subordinación. Celia es más epistemóloga y más filósofa en el sentido tradicional, y Amelia se dedica a la ética y a la filosofía política. Creo que me han influido mucho en el sentido de tener muy claro que hay unos principios a los que no se pueden renunciar como los criterios de justicia e igualdad.

Y luego una persona que me influyó muchísimo, que no me ha enseñado directamente, pero con quien he tenido un trato muy largo, incluso dimos un curso de doctorado juntas en la Universidad de las Islas Baleares, es Helen Longino. O sea, para mí, leer, en 1990 y poco, su libro Science as Social Knowledge, donde muestra el papel de los valores en la ciencia y no sólo en la ciencia mala, sino también en la buena ciencia y cómo los valores de género son determinantes, esto me encantó y fue un punto de inflexión. Es un libro que descubrí en Berkeley en el año de 1994. Creo que también me ha hecho centrarme mucho en eso. Una de las cosas en las que me centro es en el papel del género en los contenidos de la ciencia, porque sociológicamente está muy bien en presentar los números pero hay que hacer más.

El otro día me preguntaba alguien: “¿Cuál creéis tú que es la asignatura pendiente de los estudios de ciencia, tecnología y género?" Pues creo que la asignatura pendiente - y tiene que ver con lo que tú preguntabas de qué pasa en España - es que ahora la gran mayoría de las mujeres científicas de todo tipo, ciencias exactas, naturales, sociales, humanidades, se ha dado cuenta de que hay que mejorar nuestra situación. Les gusta pelear las dificultades. Todas son conscientes de que hay pocas en los altos niveles y que hay que mejorarlo. Pero, lo que todavía falta es - creo que se vio muy bien en la mesa el otro día ${ }^{22}$ con la intervención de Flora de Pablo $^{23}$ - que la gente se dé cuenta de que el género influye en todo; no sólo en las pruebas clínicas de medicina, y a lo mejor en Física Cuántica también.

Hay un físico cuántico muy importante. Un día le dije: "No sé si el género en Física Cuántica influye o no". Él me preguntó: "¿influye en Filosofía?". Yo contesté: "iClaro, muchísimo!" Él me dijo: "Pues, en Física Cuántica también. Habrá que estudiarlo". No lo he hecho. No sé si en Física... Sé que influye en el sentido de que se han pasado por alto, invisibilizado lo que han hecho muchas mujeres, pero me estoy refiriendo a contenidos. De la 
CSIC. Su campo de especialización es la biología celular y molecular con foco en la regulación génica, los receptores y las acciones de la insulina embrionaria.

${ }^{24}$ El IX Premio de Divulgación Feminista "Carmen de Burgos" fue otorgado por unanimidad al artículo de Eulalia Pérez Sedeño, en marzo de 2001 , ocasión en que ella era Catedrática de Lógica y Filosofía de la Ciencia de la Universidad del País Vasco y Presidenta de la Sociedad de Lógica, Metodología y Filosofía de la Ciencia en España. Su artículo había sido publicado en la revista Meridiam. Este premio tiene carácter anual y ámbito nacional, es convocado cada 8 de marzo por la Asociación de Estudios Históricos sobre la Mujer (AEHM), en conmemoración al Día de la Mujer y su objetivo es reconocer la labor de divulgación en el campo de la temática feminista.

${ }^{25}$ Nace en Almería, donde su padre era cónsul de Portugal y pasa su niñez y primera adolescencia en Rodalquilar, un pueblecito de la costa almeriense, donde su familia poseía una finca. Allí se inició su afición a la lectura. Después de la muerte trágica de su hijo, se divorcia de su marido y con su hija va a vivir en Madrid. En 1901, publica su primer libro: Notas del alma. Murió el 9 de octubre de 1932.

${ }^{26}$ Nacido en 1888 y casado con Luisa Sofovich, fue un gran escritor en España. misma manera, por ejemplo, que se ve en la evolución. Se ve muy claramente como el género está en Sociobiología, en Arqueología, en fin, en muchas disciplinas. No en todos los momentos, pero en muchos, o sea, cómo los sesgos de género, cómo los valores de género están dirigiendo la investigación. Como se están admitiendo determinadas justificaciones científicas como justificativas y que no lo son, y que están permeadas de género. Creo que eso falta. Eso es lo que haría falta. Es verdad que en España hay mucha gente haciendo estudios en Historia, Ciencias Sociales y Humanidades cuya especialidad es género. En Medicina hay un núcleo muy importante. Entonces creo que eso es la gran asignatura feminista todavía. A mí me parece increíble que alguien pueda dar una clase, por ejemplo de Biología Evolucionista [y no Evolutiva], y que no se dé cuenta de los sesgos de género que hay ahí. Me acuerdo que yo di durante muchísimos años en la Universidad Complutense - desde el año 90 hasta 99 - una asignatura que se llamaba "Historia de las ideas científicas sobre las mujeres". Al principio era una signatura de las que nosotros llamamos de libre configuración, que se la pone y se la acepta en cualquier carrera. Al principio venía muchísima gente de la licenciatura en Biología y ellos se quedaban alucinados de que en su carrera no se les hablara del sexismo que hay ahí implícito. Teníamos también mucha gente de Psicología y de Antropología y todas decían lo mismo. Ya cuando tenía alguien de Medicina, ellos se quedaban alucinados cuando les contaba que, en los ensayos clínicos para la eficacia de la aspirina en enfermedades coronarias, 16 mil hombres la habían probado; no había ninguna mujer.

REF: Cuéntenos sobre el premio "Carmen de Burgos"24 que ha recibido con el artículo La invisibilidad y el techo de cristal.

EPS: Yo había publicado un artículo que me fue pedido para la revista Meridiam, que es del Instituto Andaluz de la Mujer. Ellos sacaran un número especial. Era sobre algo como: "¿Qué pensamos las que hemos llegado?" Como primera y entonces única catedrática de Filosofía de la Ciencia, me dijeron que escribiera algo sobre mi experiencia. Les conté lo que cuento en el artículo. Fue muy bonito porque he sido una de las que les han concedido el premio por unanimidad. Cuando me llamaron para comunicármelo, me lo dijeron: "Nos sentimos todas muy identificadas". Estoy muy contenta porque Carmen de Burgos $^{25}$ fue una mujer muy interesante y que tuvo una vida apasionante. Una de las primeras si no la primera periodista y corresponsal de guerra de lengua española. Ella se casó con un auténtico monstruo, horrible, maltratador. Pues lo abandonó. Y se fue a Madrid y vivió una vida absolutamente libre. Fue amante de Ramón Gomez de La Serna. ${ }^{26}$ Nunca se casaron 
porque además nunca quiso casarse con él. Estamos hablando de los años 20 del siglo XX. Que era muy fuerte en la España de esa época que una mujer vivera sola, en Madrid, que había abandonado su familia etc. Ese premio es de divulgación. Ese es otro problema que hay: la divulgación no está bien vista en la academia y se supone que yo sea académica. Pero eso es otra historia.

REF: ¿Cómo ve usted los estudios de género en este contexto de España ahora en el Sistema Científico Europeo?

EPS: Creo que no se está haciendo gran cosa en Europa. Nosotros en España tenemos algo nuevo, que es que con la ley de la igualdad y la ley de la ordenación universitaria - las dos conjuntamente - dicen que hay que dar un enfoque de género a toda la enseñanza universitaria.

REF: ¿Desde cuándo es esa ley?

EPS: Desde el año pasado. Es muy reciente. Nosotros estamos intentando que en las distintas licenciaturas haya alguna asignatura de género, o se le dé enfoque de género en esas signaturas. Pero es una pelea muy difícil. En Europa creo que no se está haciendo nada.

REF: Creíamos que España estaba mejor que en otros países en esa área.

EPS: No. Creo que podríamos estar mejor si se hiciera caso de la ley. Creo, por ejemplo, que en Holanda, en los países nórdicos, ellos también tienen muy bien establecido eso. Pero creo que hay un problema, y es un problema serio que se puede dar, que es el famoso corralito, es decir que en los estudios de género se puedan quedar al margen y a la par de esto no permear las disciplinas.

REF: En Brasil el sistema que existe es muy disciplinario. La idea de la interdisciplinaridad es muchísimo importante pero en la práctica académica la interdisciplinaridad no es tan valorizada como la disciplinaridad. ¿Cómo es eso en España?

EPS: Creo que aquí pasa lo mismo. Nosotras en los años 80 teníamos la posibilidad que eso pudiera se formar en institutos de la mujer, etc. Creo que hicimos lo que había que hacer, que era intentar ocupar un lugar. Debo decir que llevo años en los estudios de género pero también en la Filosofía de la Ciencia. Pienso que son lo mismo. Es que hago Filosofía de la Ciencia o la Historia de la Ciencia con mirada de género. Desde hace tiempo - desde el año 1993 hasta al 2006 - fui presidenta de la Sociedad Española de Lógica y Filosofía de la Ciencia. Ahí, por ejemplo, introduje a los estudios de género como un tema a más. En todos los congresos metíamos ponencias sobre género. Hay un problema grave en todo esto: que nos leemos entre 
nosotras, pero luego nuestras colegas en nuestras propias disciplinas no nos leen por lo general. Salvo honrosas excepciones.

REF: ¿Cómo ve usted esta cuestión de leer y de las lenguas? ¿Aquí se publica sólo en español o también se publica en inglés?

EPS: Debo decir que me he resistido mucho. Porque creo - y de ahí proponer los congresos iberoamericanos - que los países iberoamericanos compartimos temas y problemáticas específicas. En el caso de los estudios de género tenemos mucho en común, más que con las norteamericanas. Y hay otras cosas. Por ejemplo, en las comisiones de evaluación donde he estado, hemos conseguido que lo internacional no se equiparara al inglés. Internacional es una publicación en México o en Brasil, como en Estados Unidos o en Inglaterra.

Madri, julio de 2008. 